halten, was dem Verständnisse die geringste Schwierigkeit bietet. Es wird aber und muß eine Zeit kommen, wo man einsehen wird, daß Vieles, in solchen Dingen schon gar, nicht so ist, wie man es am leichtesten begreifen könnte, während es der Eigenart des menschlichen Begriffs- und Fassungsvermögens einstweilen versagt ist, den wirklichen, realen Sachverhalt zu verstehen. Nur die Tatsachen an und für sich behalten unter allen Umständen unerschütterlich ihren unantastbaren Wert: ihnen muß der menschliche Intellekt sich unterordnen und anpassen" $\left.{ }^{1}\right)$.

\title{
Der Einfluß des Bodens auf die Blütenfüllung der Tulpen.
}

\author{
Von Karl Ortlepp in Gotha.
}

Seit fünf Jahren bin ich mit Vorarbeiten zu einer monographischen Bearbeitung der Füllungserscheinungen bei Tulpenblüten beschäftigt. Im Laufe dieser Zeit stellte ich verschiedene Kulturversuche an, um zu ergründen, ob die Tochterzwiebeln die gleiche Füllungsstärke wie die Mutterzwiebeln zeigen oder inwieweit sie von diesen abweichen, ob eine Einwirkung der angewandten Kulturmethode zu bemerken ist und in welcher Weise diese sich geltend macht. Die erste Anregung zu meinen Arbeiten über die Tulpenfüllung erhielt ich durch Prof. Goebel in München, der mir riet, meine früheren vereinzelten Beobachtungen über Tulpenfüllung fortzuführen und zu einer Monographie der Füllungserscheinungen bei Tulpen zu erweitern.

Da bis zum Abschlusse der Monographie noch längere Zeit vergehen wird, entschloß ich mich, die Resultate meiner bisherigen Kulturversuche, welche immerhin schon manchen Einblick in die Ursachen der Füllung gewähren, vorläufig im nachstehenden zu publizieren.

Leider konnte ich von den im Frühjahre 1903 aus Overveen bezogenen und genau auf ihre Füllungsweise untersuchten Tulpen, die meisten erst von 1904, manche auch erst von 1905 an nach nochmaliger Untersuchung zum Vergleiche heranziehen, weil die zur

1) „Monophyletisch oder polyphyletisch?“, pag. 137. Mitteilungen des Naturwissensch. Vereins für Steiermark, Jahrg. 1905, Heft 41. Auch auf den Artikel: „Ideales und Reales aus der Morphologie“", ibidem Jahrg. 1906, Heft 42, wäre Bedacht zu nehmen, weil da Mehreres über dieses Thema zu finden ist. 
Numerierung verwendete Tinte dazu ungeeignet war (was ich leider zu spät merkte), indem sie so verblaßte, daß sich auf den im Herbste 1903 zu den Zwiebeln gesteckten Etiketten im Frühjahre 1904 bei vielen die Nummern nicht mehr erkennen ließen. Diese Tulpen wurden 1904 während der Blüte von neuem numeriert. Da ich 1904 aus Mangel an Zeit nicht alle untersuchen konnte, numerierte ich die untersuchten und die nicht untersuchten von 1 an und setzte die Nummer der letzten, die erst 1905 von neuem beschrieben wurden, in Parenthese, z. B. (8).

Natürlich können für die vorliegende Veröffentlichung nur die Tulpen in Frage kommen, welche während des Versuches mehrmals, mindestens aber zweimal zur Blüte gelangten und untersucht werden konnten, also einen Vergleich ermöglichten. Die anderen Versuche teile ich später, wenn die betreffenden Tulpen auch mehrmals geblüht haben, mit. Viele gefüllte Tulpensorten blühen nämlich nicht jedes Jahr, besonders setzen sie in schlechtem und schwerem Boden, wie ich ihn doch zum Vergleiche auch benutzen mußte, sowie endlich, wenn sie im Sommer in der Erde gelassen werden, was des Versuchs halber ebenfalls geschehen mußte, oft ein Jahr, manchmal auch länger mit Blühen aus. Bisweilen leiden auch die Blüten durch ungünstige Witterung, so daß sie verkrüppeln. Sie werden, wenn infolge solch widriger Einflüsse ihre Entfaltung stockt, dann noch häufig von Nacktschnecken und anderen Schädlingen so stark angefressen, daß eine Feststellung der einzelnen Blütenteile unmöglich ist.

So waren gerade in diesem Jahre (1907) bei mehreren für den Vergleich besonders wichtigen Tulpen, die Knospen derart verkrüppelt und von Tieren zerfressen, daß sich die einzelnen Teile nicht mehr erkennen und zählen ließen. Dazu kommt noch, daß infolge des harten Winters mehrere Tulpenzwiebeln trotz sorgfältiger Bedeckung erfroren sind oder durch Tiere vernichtet wurden.

In vorliegender Arbeit führe ich die Tulpen unter den Nummern auf, die sie bei meinen Versuchen tragen, um Verwechslungen bei der Fortführung der Versuche zu vermeiden. Ein Vergleich der Füllung zwischen zwei in demselben Jahre in einer Mutterzwiebel ausgereiften Tochterzwiebeln untereinander ist leider nur selten möglich, da viele Tulpenzwiebeln nur je eine neue Zwiebel enthalten, und wenn mehr als eine vorhanden ist, die zweite, und bisweilen vorkommende dritte, sowie auch etwa außen an der Mutterzwiebel sitzenden Brutzwiebeln so klein sind, daß sie im nächsten Jahre nicht blühen, sondern nur ein Blatt treiben. 
Um einige Anhaltspunkte zu gewinnen, in welcher Richtung sich meine Versuche am besten zu bewegen hätten, frug ich 1902 die Züchter, von denen ich die gefüllten Tulpen bezog, nach ihren Erfahrungen und Kulturmethoden, erhieltaber leider keine Antwort. Jedenfalls fürchteten sie, daß die Konkurrenz von ihren Methoden erfahren und sich diese zunutze machen könnte.

Ich mußte also zunächst durch meine Versuche feststellen, ob überhaupt den Kulturmethoden, im besonderen den verschiedenen Bodenarten èin Einfluß auf die Füllung der Tulpenblüten zukommt und schon bei geringeren physikalischen und chemischen Verschiedenheiten der Erde eine Schwankung in der Füllungsstärke zu bemerken ist. Ich arbeitete deshalb anfänglich hauptsächlich mit ganz einfachen Kulturmethoden, indem die Tulpen auf Beete im freien Lande, deren Erde von verschiedener Nährkraft und physikalischer Beschaffenheit war, gepflanzt wurden. Erst später nahm ich, zunächst nur in geringerem Umfange, Versuche mit Topfkultur und künstlicher Düngung vor. Die Erde im Garten ist schwerer Lehmboden, sehr bündig, der da, wo er selten gelockert wird, schwer zu bearbeiten ist, die Nässe sehr lange anhält, aber durch häufiges Umgraben und Kompostdüngung zu einem guten, nährstoffreichen, mittelschweren Boden wird.

Die von mir benutzten Beete in diesem Garten will ich hier nach der Beschaffenheit, die ihre Erde durch Umgraben und Düngen, oder vorangegangene Pflanzung allmählich bis zum Beginn meiner TulpenVersuche erhalten hat, kurz charakterisieren und um Wiederholungen zu vermeiden mit Buchstaben bezeichnen, unter welchen die Beete bei den Tulpen genannt sind.

Beet A. Mittelschwere Erde von sehr guter Nährkraft, da fast jährlich mit Kompost gedüngt. Knollenbegonien gediehen darauf sehr gut.

Beet B. Schwere Erde, die aber nicht von klosiger Beschaffenheit ist, mit mittelguter Nährkraft, da nur selten Kompost untergegraben wurde.

Beet $\mathrm{C}^{1}$ ). Schwere Erde 1-2 mal jährlich, aber nur oberflächlich gelockert, die keinen Kompost erhalten hat.

Beet $\mathrm{D}^{1}$ ). Schwere Erde nur 1 mal im Jahre oberflächlich gelockert und nur öfter Ruß und etwas leichte Erde darauf getan, das

1) Bei Beet C und D ist die obere Erdschicht durch jahrelanges Eingraben von Fichtennadeln etwas gelockert und trockner als bei $\mathrm{E}$ und $\mathrm{F}$. 
Beet wird aber durch Schößlinge benachbarter Sträucher etwas ausgesogen und steht deshalb nicht in guter Nährkraft.

Beet E. Schwere, sehr feuchte Erde, die durch vorangegangene Kultur nährstoffarm und dann im Frühjahre 1903 nur mit kalkarmer Komposterde gedüngt worden war, so daß 1903 vor der Tulpenkultur Fuchsien nicht gediehen, sondern ein dürftiges Aussehen zeigten.

Beet F. Schwere, feuchte Erde noch sehr mit Wurzelresten verwilderter Rosen, die früher darauf standen, durchsetzt. Pelargonien, die im Sommer 1903 hier ausgepflanzt waren, sahen krank aus.

Ich brachte auf Beet A im Herbst 1904 eine $20 \mathrm{~cm}$ hohe Schicht Komposterde und 1 Pfd. Ätzkalk und grub beides unter. 1906 wurde im Herbst wieder, aber nur eine $4 \mathrm{~cm}$ hohe Schicht und zwar weniger kräftiger Komposterde daraufgetan und eingegraben.

Die Beete C und D erhielten im Mai 1904, während der Blüte, Kohlenasche, die ich im Laufe des Winters 1903-1904 aus einem bestimmten Ofen auf einem Haufen im Freien gesammelt hatte, also ihre Zusammensetzung nach dem darin verbrannten Material ungefähr beurteilen konnte. Danach bestand diese Asche aus 3/4 Braunkohlenund 1/4 Steinkohlenasche und unbedeutenden, nur vom Anzünden herrührenden Beimengungen von Holzasche, und war, da erst im Winter vorher gesammelt, ziemlich frisch. Ich verteilte die Asche auf der Oberfläche beider Beete $5 \mathrm{~cm}$ hoch und vermischte sie dann fast spatenstichtief mit dem Boden. Die Zwiebeln blieben alle mit Ausnahme von IIa, während der Ruheperiode in der Erde. 1906 wandte ich abermals Kohlenasche an, die aber über ein Jahr gelagert hatte, also nicht mehr so scharf war, sie wurde zwar im April ausgestreut, aber erst im Juli z. T. untergegraben. Diesmal wurde aber nur Beet $\mathrm{C}$ ganz damit bestreut, von Beet $\mathrm{D}$ dagegen nur ein Teil, das durch Bretter abgetrennte Stück, auf dem X und XVII standen, bekam nichts. Auch wurde die Asche auf diesem Beete gar nicht, und auf dem Beete $\mathrm{C}$ erst dann untergegraben, nachdem die Pflanzen abgewelkt und die neuen Zwiebeln ausgereift waren. Ich nahm die Zwiebeln vor diesem Umgraben des Beetes heraus und setzte die Tochterzwiebeln dann gleich wieder ein.

Aus Beet F. entfernte ich im Herbst 1905, vorm Einsetzen der Zwiebeln, die noch bis dahin in der Erde befindlichen Rosenwurzeln, was nebst der damit verbundenen gründlichen Lockerung den Boden in physikalischer Hinsicht etwas verbesserte. Eine Düngung bekam dieses Beet jedoch nicht. 
Da es sich in der vorliegenden Abhandlung nur um einen Vergleich in der Füllung, und ob diese ab- oder zunahm, handelt, brauchen nicht alle Änderungen in der Blüte angegeben zu werden, es genügt vielmehr, wenn nur die für die Stärke der Blütenfüllung in Betracht kommenden Blütenteile angegeben werden. Es sind dies die Petala, die in fast allen gefüllten Tulpenblüten vorhandenen Übergangsgebilde zwischen den Stamina und Petalen, die bisweilen vorkommenden Übergangsformen von Carpellen zu Petalen und die Zahl der Stamina. Ich habe es nun im folgenden so eingerichtet, daf die überzähligen Petala zu den normalerweise bei der ungefüllten Blüte vorhandenen 6 hinzugezählt und alle mit $\mathrm{p}$ bezeichnet werden. Heißt es also beispielsweise $8 \mathrm{p}$, so wird damit ausgedrückt, daß die betreffende Tulpe 2 überzählige Petala besitzt. Die Übergangsgebilde, d. h. die petaloid ausgewachsenen Stamina oder Carpelle werden mit $\mu$ bezeichnet und um zugleich anzudeuten, ob die genannten Blütenteile beiderseitig oder nur einseitig petaloid ausgewachsen sind, im ersten Falle $T$, im zweiten $\Gamma$ dahintergesetzt; $2 \mu \mathrm{T}$ heißt also, daß zwei beiderseitig, $2 \mu \Gamma$, daß zwei einseitig petaloid entwickelte Übergangsgebilde in der Blüte sind. Wenn solche Übergangsgebilde alle oder zum Teil dem Anscheine nach aus Fruchtblättern hervorgegangen sind, so wird dies durch ein in Parenthese dahinter gesetztes carp., z. B. $4 \mu \mathrm{T}$ (carp.) oder $4 \mu \mathrm{T}(2$ carp.) angedeutet und heißt, daß im ersten Falle alle 4, im letzten nur 2 aus Carpellen entstanden waren.

Bei den Stamina hielt ich es für notwendig, auch die mit dem Fruchtknoten ganz verwachsenen, sowie die fadenförmig verkümmerten anzugeben, da diese öfter im nächsten Jahre wieder als freie resp. normale erscheinen oder freie resp. normale im folgenden Jahre fadenförmig verkümmert oder fast bis zur Unkenntlichkeit mit dem Fruchtknoten verwachsen sein können. 5 st (3 verkümmert) heißt, daß von den 5 Stamina nur 2 normal und bei 3 die Anthere mehr oder weniger kümmerlich entwickelt ist. 5 st (3 angew.) bedeutet, daß 2 normal und 3 an den Fruchtknoten oder freie Carpelle. in Ausnahmefällen auch Übergangsgebilde angewachsen sind. 8 st (3 verk., 2 angew.) drückt aus, daß 3 Stamina normal, 3 verkümmert und 2 angewachsen sind.

Meine Versuche ergaben bisher, daß die Art der Kultur, im besonderen aber die Bodenbeschaffenheit einen wesentlichen Einfluß auf $\mathrm{Zu}$ - oder Abnahme der Blütenfüllung bei den Tulpen ausübt.

So fand fast stets eine Zunahme in der Füllung der Tulpen statt, wenn die Mutterzwiebeln in dem guten Boden von Beet A., dessen 
Erde 1904, wie oben angegeben, noch bedeutend verbessert wurde, gestanden hatten, wie man aus den nachstehenden Beispielen ersieht.

Nr. 21.

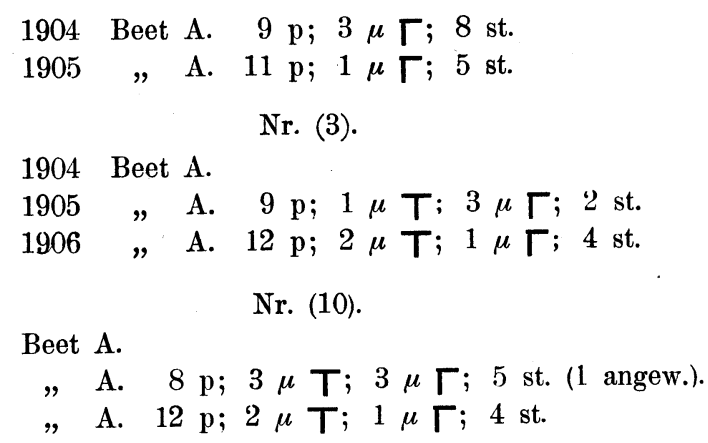

Einige Tulpen (Nr. 8, 4 und 20) zeigten in dem einen Jahre, trotzdem sie 1904 auf dem Beete A gestanden hatten, einen Stillstand resp. Rückgang in der Füllung, diese nahm aber 1906, bei Nr. 8 auch 1907, nachdem die im Herbst 1904 erfolgte Komposterde- und KalkDüngung auf die Zwiebeln 1905 eingewirkt hatte, wieder zu, was aus folgendem ersichtlich ist:

Nr. 8.

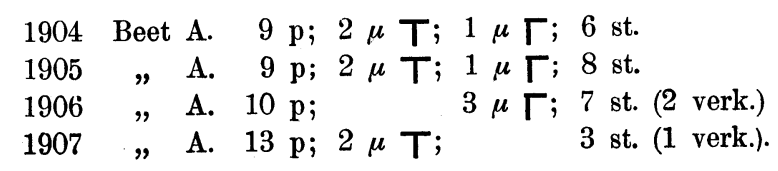

Nr. 4.

1904 Beet A. $9 \mathrm{p} ; 1 \mu$ T; $3 \mu \Gamma ; 6$ st.

$1905 \quad$ A. $9 \mathrm{p} ; \quad 3 \mu \Gamma ; 5$ st.

$1906 \Longrightarrow$ A. $13 \mathrm{p} ; 1 \mu \mathrm{T} ; 1 \mu \Gamma ; 4$ st.

1907 А. Knospe verkrüppelt und zerfressen.

Nr. 20.

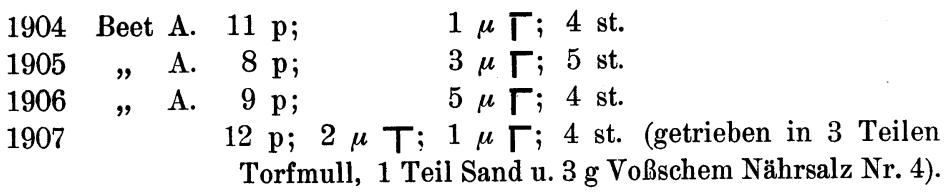

Es gelang mir ferner, durch reichliche Düngung mit Kohlenasche eine wesentliche Zunahme der Füllung zu erreichen. Die Beschaffenheit der dazu verwendeten Asche habe ich oben angegeben. Es ergibt sich aus den Beispielen, daß nur die Aschedüngung 1904 einen Erfolg 
erkennen läßt, was erstens davon herrühren kann, daß 1904 die Asche sofort und nicht erst nach Eintritt der Ruheperiode untergegraben wurde, so daß sie noch auf die Pflanzen 1904 einwirkte, zweitens dürfte aber auch die weniger abgelagerte Asche mehr zur Bodenlockerung, Aufschließung und Drainierung beigetragen haben.

Die günstige Wirkung der Kohlenaschedüngung von 1904 wird aus den nachstehenden Beispielen klar, denn vor der Kohlenaschedüngung hatte dir Füllung abgenommen und nahm im Jahre nach der Düngung wieder zu. Später erfolgte dann abermals eine Abnahme und solche Tulpen, bei denen der Vergleich nur vor (Nr. XVII.) (Nr. IV.) der Aschedüngung stattfand, zeigten bloß Abnahme.

\section{Nr. III a.}

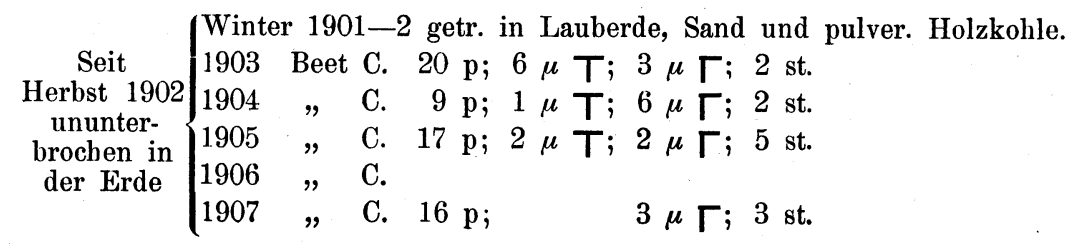

Nr. I.

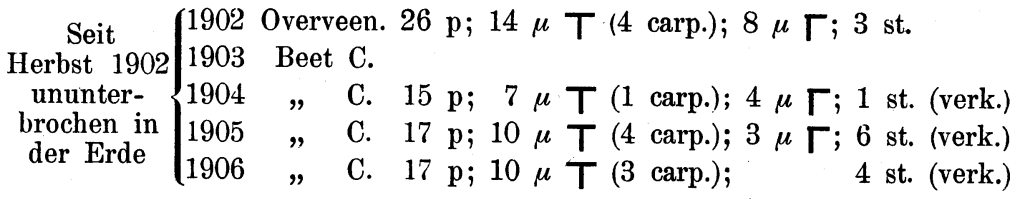

Nr. II.

Seit
Herbst $1902(1902$ Overveen. $36 \mathrm{p} ; 8 \mu \mathrm{T}(2$ carp. $) ; 9 \mu \Gamma(4$ carp. $) ; 1$ st. ununter- 1903 Beet C.

brochen in
der Erde $1904 \quad$ C. $17 \mathrm{p} ; 10 \mu \mathrm{T}(6$ carp. $) ; 3 \mu \Gamma ; \quad 1$ st.

Nach der Blüte im Sommer 1904 herausgenommen. Kleinere gleich wieder eingepflanzt, größere Tochterzwiebel II a erst im Herbst eingesetzt.

Nr. II a.

1905 Beet C. $29 \mathrm{p} ; 5 \mu$ 丁; $\quad 3 \mu \Gamma$ (1 carp.)

Nr. X.

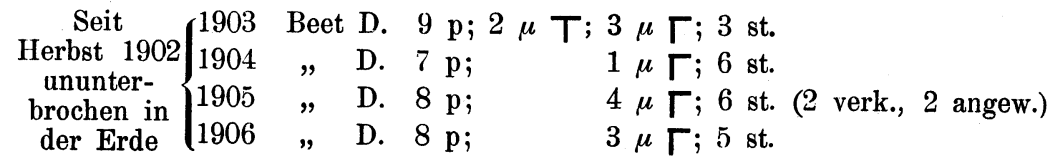




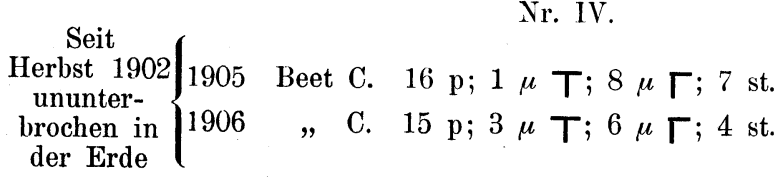

Nr. XVII.

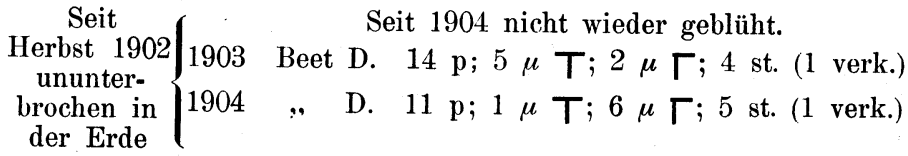

Eine Abnahme in der Füllung trat regelmäßig bei den Tulpen ein, deren Mutterzwiebeln in schlechter, besonders sehr schwerer Erde gestanden hatten.

Nr. (0).

1904 Beet B. Blüte verkrüppelt

$1905 \quad$ F, $10 \mathrm{p} ; 5 \mu$ T; $2 \mu \Gamma ; 4$ st.

$1906 \quad$ F. $9 \mathrm{p} ; \quad 2 \mu \Gamma ; 7$ st.

1907 „ A. Blüte verkrüppelt.

1904 Beet A.

$1905 \quad$ \% F. 9 p; $2 \mu$ T; $5 \mu \Gamma ; 6$ st.

1906 ” A. $9 \mathrm{p} ; 2 \mu$ T; $4 \mu \Gamma ; 5$ st.

Nr. (6).

1904 Beet A.

$1905 \quad$ \%. $14 \mathrm{p} ; 5 \mu$ T; 6 st.

1906 " F. $10 \mathrm{p} ; 3 \mu$ T; $4 \mu \Gamma ; 3$ st.

Nr. 10.

1904 Beet A.

$1905 \quad$ F. $10 \mathrm{p} ; \quad 3 \mu \Gamma ; 3$ st

$190610 \mathrm{p} ; \quad 1 \mu \Gamma ; 7$ st. (getrieben in $1 \frac{1}{2}$

Teil Asche, $1 \frac{1}{2}$ T. Heideerde und 1 T. Sand).

Nr. 3.

1904 Beet A.

$1905 \quad$ F. $9 \mathrm{p} ; 5 \mu$ 丁; $2 \mu \Gamma ; 1$ st.

$1906 \quad 9 \mathrm{p} ; 3 \mu \mathrm{T} ; 4 \mu \Gamma$ (getrieben in $1 \frac{1}{2}$ Teil.

Lauberde, $1 \frac{1 / 2}{2}$ T. Asche u. 1 Teil Sand).

Aus den Versuchen mit IIIa, I, II, X, IV und XVII ist außerdem noch zu ersehen, daß auch die Behandlung der Tulpe nach der Blüte einen Einfluß auf die Füllungsstärke der nächstjährigen Blüte aus- 
übt. Läßt man nämlich die Zwiebel in der Erde, so wird die Füllung im folgenden Jahre eine Abnahme zeigen, wenn nicht durch geeignete, hier Kohlenaschedüngung, dem vorgebeugt wurde. Nimmt man dagegen die Pflanze nach dem Welken des Laubes aus der Erde, löst die Tochterzwiebel heraus und hebt sie trocken bis zum Einsetzen im Herbste auf, so wird, falls die Tulpe nicht in schlechtem Boden stand, die nächstjährige Blüte noch gerade so stark, eher stärker gefüllt sein. Diese Zunahme kann unter besonders günstigen Umständen, wie nach einer Aschedüngung, siehe II a, sògar sehr bedeutend werden.

Es machte sich sogar dann noch ein günstiger Einfluß der trockenen Aufbewahrung geltend, wenn infolge derselben eine Generation nicht zur Entfaltung gelangte. Im Herbst 1902 konnte ich verschiedener Umstände wegen nicht alle Tulpenzwiebeln einsetzen. Im Frühjahr 1903 hatten die Zwiebeln, trotzdem sie in einem kühlen Raume aufbewahrt wurden, einige Zentimeter lang ausgetrieben. Diese Triebe welkten bald, worauf ich die Tochterzwiebeln herauslöste. Im Herbst 1903 pflanzte ich diese auf verschiedene Beete. Bei der Blüte 1904 zeigten, wie man aus der folgenden Wiedergabe meiner Versuche ersehen wird, Nr. 75, 77 und 96 eine nicht unwesentliche Zunahme in der Füllung und nur bei 87 und 76 hatte sie abgenommen, aber bei der letzten war die Abnahme ganz unbedeutend. Immerhin ist also selbst diese unnatürlich lange trockene Aufbewahrung eher von förderndem als hemmendem Einflusse auf die Füllung gewesen.

Nr. 75 .

1902 Overveen. $7 \mathrm{p} ;$
1903 trocken aufbewahrt
1904
Beet B. $\quad 9 \mathrm{p} ; 5 \mu \mathrm{T} ; 1 \mu \Gamma ; 2$ st. (1 verk.).

Nr. 77.

1902 Overveen. $6 \mathrm{p} ; 1 \mu \mathrm{T} ; 5 \mu \Gamma ; 4$ st.

1903 trocken aufbewahrt

1904 Beet B. $8 \mathrm{p} ; 6 \mu \mathrm{T}$; 2 st. (1 angew.)

$1905 \quad$ F.

1906 " $\quad$ F. $8 \mathrm{p} ; 1 \mu$ T; $3 \mu \Gamma ; 2$ st.

1907 А. $7 \mathrm{p} ; .4 \mu \Gamma ; 4$ st.

Nr. 96.

1902 Overveen. $24 \mathrm{p} ; 4 \mu \mathrm{T} ; 4 \mu \Gamma ; 4$ st.

1903 trocken aufbewahrt

1904 Beet D. $29 \mathrm{p} ; 1 \mu$ T; $1 \mu \Gamma ; 2$ st. (1 angew.). 
Nr. 76 .

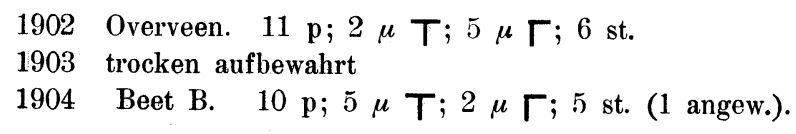

Nr. 87.

1902 Overveen. $10 \mathrm{p} ; 5 \mu \mathrm{T} ; 2 \mu \Gamma ; 2$ st.

1903 trocken aufbewahrt

1904 Beet B. $8 \mathrm{p} ; 5 \mu \mathrm{T}$; 3 st.

Während die ersten Beispiele in dieser Arbeit zeigen, wie bei fortgesetzter Kultur in guter Erde, Beet A, die Füllung immer mehr zu, und bei Kultur in schlechter Erde, Beet F, abnimmt, sehen wir an Nr. 77, die durch mehrere Jahre auf verschiedene Weise kultiviert wurde, dementsprechend die Füllung zu- und wieder abnehmen. Auch die folgenden auf verschiedenen Beeten kultivierten Tulpen bestätigen meine Ansicht von der Einwirkung des Bodens. Man sieht an ihnen auch, daß die Abnahme weniger groß bei Beet $B$ und $E$, sondern am sichersten und größten ist nach Kultur, besonders mehrjähriger auf Beet F, dessen Erde am schlechtesten ist. Die Abnahme zeigt sich auf diesem Beete am augenfälligsten, bevor die Rosenwurzeln daraus -entfernt und es dadurch verbessert wurde. Hatten dagegen die Zwiebeln vorher auf besseren Beeten gestanden (Nr. 77) und Nr. (6) 1906 so war die schädliche Einwirkung des Beetes F nicht so groß. Außerdem ist an den folgenden Beispielen auch die Einwirkung verschiedener Erdarten bei Topfkultur und mehrerer Düngesalze ${ }^{1}$ ) zu erkennen.

Nr. 11.

1904 Beet A. 9 p; $3 \mu$ T; $2 \mu \Gamma ; 3$ st.

$190510 \mathrm{p} ; 5 \mu \mathrm{T} ; 2 \mu \Gamma ; 1$ st. (getrieben in 4 Teilen Lauberde, 1 Teil Sand und einer Hand voll Holzkohlenpulver und $2 \mathrm{~g}$ Kalkstickstoff.

Nr. 28.

1904 Beet A. 13 p; $2 \mu$ T; 1 st.

$190514 \mathrm{p} ; 2 \mu$ T; 3 st. (1 angew.) (getrieben in 2 Teilen Torfmull, 2 Teil. Lauberde, 1 Teil Sand, einer Hand voll Holzkohlenpulver, $1 \mathrm{~g}$ Chilisalpeter.

No. 22.

1904 Beet A. $13 \mathrm{p} ; 4 \mu$ T; 3 st. (1 angew.)

1905 " F. $11 \mathrm{p} ; 6 \mu$ T; 3 st. (1 angew.)

1) Die Düngesalze wurden bei allen Versuchen vorm Einsetzen der Zwiebeln, der Erde in Pulverform beigemischt. 
Nr. 22 a.

1906

$10 \mathrm{p} ; 3 \mu \mathrm{T} ; 2 \mu \Gamma ; 2$ st. (1 angew.) (getrieben in $1 \frac{1}{2}$ Teil. kalkreicher Kompost-, $1 \frac{1}{2}$ Teil. Mistbeeterde, 1 Teil Sand und $63 / 4 \mathrm{~g}$ Thomasmehl.

Nr. 22 b.

1906 Beet D. $11 \mathrm{p} ; 3 \mu$ T; $3 \mu \Gamma ; 1$ st.

Nr. 30

1903 Overveen. $9 \mathrm{p} ; \quad 3 \mu \Gamma ; 5$ st.

1904 Beet A. $8 \mathrm{p} ; 3 \mu$ T; $3 \mu \Gamma ; 2$ st.

1905

$8 \mathrm{p} ; 2 \mu \mathrm{T} ; 1 \mu \Gamma ; 4$ st. (getrieben in 1 Teil kalkreicher Kompost-, 1 Teil Lauberde, 1 Teil Sand und $1 / 5$ Teil Holzkohlenpulver.

Nr. 32.

1904 Beet E. Blüte zerfressen.

$1905 \quad$ A. 12 p; $3 \mu$ T: $8 \mu \Gamma ; 4$ st. (2 zu Faden verk.)

$1906 \quad 15 \mathrm{p} ; 1 \mu \mathrm{1} ; 4 \mu \Gamma ; 6$ st. (getrieben in 3 Teilen

Lauberde und 1 Teil Sand.

Nr. 55.

1904 Beet E. $8 \mathrm{p} ; \quad 4 \mu \Gamma ; 3$ st.

$1905 \quad$ F. $9 \mathrm{p} ; \quad 2 \mu \Gamma ; 9$ st.

Nr. 13 (alt numeriert).

1903 Overveen. Blüte stark verschimmelt, deshalb nicht untersuchen können.

1904 Beet A. $10 \mathrm{p} ; 5 \mu \mathrm{T} ; 1 \mu \Gamma ; 1$ st. (angew.)

$190513 \mathrm{p} ; 2 \mu \mathrm{T} ; 1 \mu \Gamma ; 2$ st. (1 angew.) (getrieben in Heide- und Lauberde mit Sand, $1 \mathrm{~g}$ Chilisalpeter.

$19068 \mathrm{p} ; 3 \mu \mathrm{T} ; 2 \mu \Gamma$ (getrieb. in 1 Teil Komposterde, 1 Teil Lauberde, 1 Teil Sand u. 5 g Kalkstickstoff.

1904 Beet A.

Nr. (4).

1905

, A. $10 \mathrm{p} ; 1 \mu \mathrm{T} ; 5 \mu \Gamma ; 5$ st.

$1906 \quad 10 \mathrm{p} ; \quad 6 \mu \Gamma ; 5$ st. (getrieben in $1 \frac{1}{2}$ Teilen kalkreicher Kompost-, $1 \frac{1}{2}$ Teilen Mistbeeterde und 1 Teil Sand.

1904 Beet A.

Nr. (8).

$1905 \quad$ F. $13 \mathrm{p} ; 1 \mu \mathrm{T} ; 5 \mu \Gamma ; 6$ st. (1 angew.)

1906 \%. $13 \mathrm{p} ; 1 \mu$ T; $5 \mu \Gamma ; 1$ st.

Nr. 80.

1902 Overveen. $16 \mathrm{p} ; 2 \mu \mathrm{T} ; 9 \mu \Gamma ; 5$ st.

1903 trocken aufbewahrt.

1904 Beet A.

1905
" F. $18 \mathrm{p} ; 2 \mu$ T; $4 \mu$ Г $; 5$ st. 
Nr. 83.

1902 Overveen. $18 \mathrm{p} ; 1 \mu \mathrm{T} ; 4 \mu \Gamma ; 7$ st.

1903 trocken aufbewahrt.

1904 Beet B. Blüte verkrüppelt.

$1905 \quad$ A. $11 \mathrm{p} ; 1 \mu$ T; $6 \mu \Gamma ; 4$ st.

Nr. 97 e.

1902 Overveen. $8 \mathrm{p} ; 5 \mu \mathrm{T} ; 2 \mu \Gamma ; 3$ st.

1903 trocken aufbewahrt.

1904 Beet E.

$1905 \quad$ F. $11 \mathrm{p} ; \quad 6 \mu \Gamma ; 6$ st.

Nr. 97 e, a.

1906

$11 \mathrm{p} ; \quad 1 \mu \Gamma ; 7$ st. (getrieben in 3 Teilen kalkreicher Komposterde, 1 Teil Torfmull, 1 Teil Sand, 5 g Wag. Nährsalz.

Nr. 97 c.

1902 Overveen. $8 \mathrm{p} ; 5 \mu \mathrm{T} ; 2 \mu \Gamma ; 3$ st.

1903 trocken aufbewahrt.

1904 Beet A.

$1905, \quad$ F.

$1906 \quad$ А. $7 \mathrm{p} ; 1 \mu$ Т; $3 \mu$ \%; 5 st.

Im folgenden gebe ich eine Reihe Versuche wieder, in denen es sich nur um Veränderungen handelt, welche die aus Overveen blühend bezogenen Tulpen im ersten Jahre ihrer Kultur bei mir zeigten. Mit Ausnahme der beiden ersten Beispiele von früher eingeführten waren folgende Tulpen $1906 \mathrm{zu}$ gleicher Zeit aus Holland bezogen, hatten also alle dieselbe Vorkultur und wurden dann im Herbst 1906 in verschiedene Beete und in Töpfe mit besonderen Erdmischungen und Düngungen gepflanzt. Dabei zeigte sich, daß die aus Overveen stammenden Zwiebeln im nächsten Jahre meist zur Zunahme in der Füllung neigen, was jedenfalls auf den günstigen Einfluß der offenbar sehr lockeren, guten Erde zurückzuführen ist.

Nr. 42.

1903 Overveen. $9 \mathrm{p}$;

1904 Beet A. $13 \mathrm{p} ; 2 \mu \mathrm{T}$; $2 \mu \Gamma ; 7$ st.

6 st.

Nr. 23.

1903 Overveen. $18 \mathrm{p} ; \quad 15 \mu \Gamma ; 2$ st.

$190425 \mathrm{p}$ (alle schmal) 3 st. (2 verk.) (getrieben auf Wasser mit 1 g Wag. Nährsalz. Zw. kaputt). 
Nr. 107.

1906 Overveen. $11 \mathrm{p} ; 4 \mu$ T; $6 \mu \Gamma ; 5$ st.

1907 Beet A. $18 \mathrm{p} ; 4 \mu$ T; $5 \mu \Gamma ; 4$ st.

Nr. 113.

1906 Overveen. $20 \mathrm{p} ; 2 \mu$ T; $6 \mu$ Г; 5 st.

1907 Beet A. $19 \mathrm{p} ; 9 \mu$ T; $3 \mu \Gamma$ (carp.); 6 st.

Nr. 115

1906 Overveen. $15 \mathrm{p} ; 6 \mu \mathrm{T} ; 2 \mu \Gamma ; 6$ st.

1907

$16 \mathrm{p} ; 4 \mu \mathrm{T} ; 9 \mu \Gamma ; 9$ st. (getrieben in 3 Teilen Torfmull und 1 Teil Sand; $1 \mathrm{~g}$ schwefelsaures Ammoniak, $1 \mathrm{~g}$ schwefelsaures Kali und $1 \mathrm{~g}$ Superphosphat.

Nr. 116

1906 Overveen. $9 \mathrm{p} ; 2 \mu \mathrm{T} ; 1 \mu \Gamma ; 7$ st.

$1907 \quad 10 \mathrm{p} ; 1 \mu$ T; $3 \mu \Gamma ; 3$ st. (getrieben in 3 Teilen Torfmull, 1 Teil Sand, $1 \mathrm{~g}$ schwefels. Amm.).

Nr. 117.

1906 Overveen. $15 \mathrm{p} ; \quad 2 \mu \Gamma ; 7$ st.

1907 Beet A. $14 \mathrm{p} ; 2 \mu \mathrm{T} ; \quad 4$ st.

Nr. 118.

1906 Overveen. $13 \mathrm{p} ; \quad 2 \mu \Gamma ; 7$ st.

1907 Beet A. $\quad 16 \mathrm{p} ; \quad 4 \mu \Gamma ; 1$ st.

Nr. 122.

1906 Overveen. $36 \mathrm{p} ; \quad 4 \mu \Gamma ; 8$ st.

$190740 \mathrm{p} ; 3 \mu \mathrm{T} ; 4 \mu \Gamma ; 7$ st. (getrieben in 3 Teilen Torfmull, 1 Teil Sand).

Nr. 123.

1906 Overveen. $35 \mathrm{p} ; 3 \mu \mathrm{T} ; 5 \mu \Gamma ; 7$ st.

1907 Beet A. $43 \mathrm{p} ; 2 \mu$ T; $2 \mu \Gamma ; 6$ st.

Nr. 125 .

1906 Overveen. $34 \mathrm{p} ; 2 \mu \mathrm{T} ; 3 \mu \Gamma ; 5$ st.

1907 Beet A. $\quad 40 \mathrm{p} ; 7 \mu$ T; $2 \mu \Gamma ; 11$ st. (4 angew. 2 verk.).

Nr. 127.

1906 Overveen. $11 \mathrm{p} ; 2 \mu \mathrm{T} ; 4 \mu \Gamma ; 4$ st.

$19078 \mathrm{p} ; 6 \mu$ T; $1 \mu \Gamma$ (getrieben in 3 Teilen Torfmull, 1 Teil Sand und $1 \mathrm{~g}$ Chilisalpeter).

Nr. 130.

1906 Overveen. $10 \mathrm{p} ; \quad 4 \mu \Gamma ; 4$ st.

1907 Beet F. $19 \mathrm{p} ; 7 \mu \mathrm{T}$ (Petale klein). 
Nr. 134.

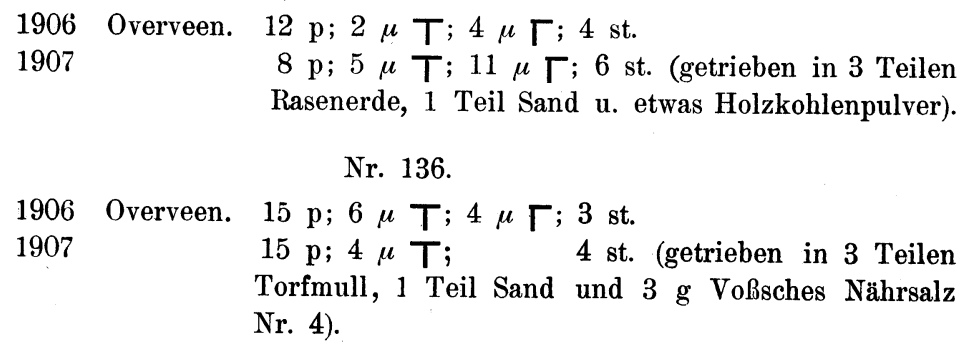

Aus den im vorstehenden mitgeteilten Versuchen ersieht man, daß die Stärke der Blütenfüllung bei den Tulpen vor allem davon abhängt, welche Beschaffenheit die Erde hat, in der die Tulpen kultiviert werden. Da sich der günstige oder ungünstige Einfluß erst im folgenden Jahre zeigt, muß die Erde schon auf die Mutterzwiebel in der Weise einwirken, daß die in ihr ausreifende Tochterzwiebel eine Tendenz zur Zu- oder Abnahme der Füllung erhält. Außer dieser indirekten Einwirkung, die der neuen $Z$ wiebel gleich gewisse Qualitäten mitgibt, scheint die Beschaffenheit der Erde noch eine, wenn auch viel schwächere direkte Wirkung auf die Blütenfüllung der fertigen, austreibenden Zwiebel auszuüben. Darauf sind manche der scheinbaren Ausnahmen zurückzuführen, indem die offenbar schon vorhandene Tendenz zur Zunahme, z. B. bei Nr. (3), (10), 8, oder zur Abnahme, z. B. Nr. (0), durch gute oder durch schlechte Erde gesteigert wurde, resp. die der Zwiebel innewohnende Neigung zur Zunahme bei Nr. 22 im Jahre 1905 vor der Verbesserung des Beetes F. sich nicht entfalten konnte, weil besonders widrige Verhältnisse entgegenwirkten, so daß sogar eine Abnahme eintrat oder die Neigung zur Abnahme durch besonders gute Erde fast aufgehoben wurde, wie z. B. bei Nr. (1).

Diese direkte Wirkung auf die Füllung tritt so recht deutlich zutage bei der diesjährigen (1907) Blüte der erst im vorigen Jahre blühend aus Overveen bezogenen Tulpen, welche doch nach gleicher oder annähernd gleicher Kultur meist eine Zunahme, die auf günstige dortige Bedingungen zurückzuführen ist, zeigen, aber auch bei einzelnen eine Abnahme erkennen lassen, welche jedenfalls durch direkte Einwirkung ungünstiger Verhältnisse entstand, wie andererseits auch die Zunahme in der Füllung mancher Tulpen noch durch direkte Wirkung günstiger Umstände gesteigert wurde.

$\mathrm{Da}$ ich die mir zur Verfügung für meine Tulpenversuche stehenden Beete zum Vergleiche der verschiedenen Bodenwirkung und der Aschedüngung brauchte, war ich mit den Nährsalzversuchen auf Gefäßkultur angewiesen. Wenn man die Töpfe mit Tulpen aber im Keller über- 
wintert $^{1}$ ), so wachsen die Triebe im Dezember oder Januar schon so hoch, daß man die Tulpen, um ein Vergeilen zu verhüten, ins Zimmer bringen muß, wodurch sie getrieben werden, trotzdem man dies eigentlich nicht wollte. Um nun hier einen Ausweg zu schaffen und Nährsalzdüngung-anzuwenden ohne die Tulpen $\mathrm{zu}$ treiben und so einen Vergleich mit durch Nährsalze gedüngten Treibtulpen einerseits und nicht mit Nährsalz gedüngten Freilandtulpen anderseits zu haben, pflanzte ich vergangenes Jahr eine Anzahl Tulpenzwiebeln in Steinkasten, die ich mit verschiedenen Düngungen versah. Nach dem Bepflanzen wurden die Kasten bis zum Rande ins Land eingesetzt, fest mit Erde umgeben und gut mit Fichtenreisig bedeckt. Trotz der guten Bedeckung gingen aber in diesem ungünstigen Winter fast alle Tulpen in den Kästen zu Grunde und die wenigen übrig gebliebenen blühten nicht. So war ich also auch dieses Mal wieder bei der Prüfung der Nährsalzwirkung nur auf die in Töpfen befindlichen, erst im Keller und dann in der Stube stehenden Tulpen beschränkt.

Der direkte Einfluß der Topferde beim Treiben auf die Stärke der Füllung läßt sich noch nicht recht übersehen, immerhin scheint aber auch hier eine leichte, lockere Erde der Füllung günstiger zu sein als eine schwere, bündige. Bei Nr. 32 in der Lauberde- und Sandmischung, war zwar die Zahl der Übergangsgebilde um 6 Stück geringer als im Jahre vorher, dafür aber die Petala um 3 vermehrt, so daß die Füllung wohl als gleichgeblieben angesehen werden kann. Trotzdem diese Tulpe also 2 Jahre vorher auf Beet $\mathrm{E}$ stand, hatte die Füllungsstärke nicht abgenommen. Von den beiden in Aschemischung getriebenen Tulpen, die das Jahr vorher auf Beet $\mathrm{F}$ und zwei Jahre früher auf Beet A standen, zeigte Nr. 10 in der Asche-HeideerdeMischung $2 \mu \Gamma$ weniger, Nr. 3 in der Asche-Lauberde-Mischung hatte der Zahl nach gar nicht, sondern nur insofern etwas abgenommen, als $2 \mu T$ in $2 \mu \Gamma$ zurückgegangen waren. In der Mischung von Torfmull, Sand und Holzkohlenpulver zeigte Nr. 122, sowohl der Zahl als auch der Entwicklung nach, $-4 \mathrm{p}$ und $3 \mu \mathrm{T}$ mehr, eine ziemlich bedeutende Zunahme, die aber zum Teil wohl noch auf die Kultur in Overveen zurückzuführen ist, wobei man erwägen muß, daß die in Asche getriebenen das Jahr vorher in Beet $\mathrm{F}$ gestanden hatten.

Dagegen ergaben von den Tulpen in schwerer Erde, Nr. 30 in kalkreicher, Kompost-, Lauberde, Sand und Holzkohlenpulver eine deutliche Abnahme, obwohl sie das Jahr vorher auf Beet A gewesen, aller-

1) In Erdgruben wollte ich sie nicht überwintern, weil die Blumenzwiebeln hier leicht von Mäusen angefressen werden. 
dings auch hier nur wenig Zunahme nach der Kultur in Overveen zu bemerken war. Bei Nr. 134 in Rasenerde, Sand und Holzkohlenpulver nahm die Füllung trotz der günstigen Kultur in Overveen nur der Gesamtzahl nach zu, aber die Anzahl der Petala war zurückgegangen und eine Vermehrung hatte vor allem nur in $\mu \Gamma$ stattgefunden. Bei Nr. (4), die ich in kalkreicher Kompost-,Mistbeeterde und Sand trieb war nur $1 \mu \mathrm{T}$ in $\mu \Gamma$ reduziert. Hier hatten wohl teils die inneren günstigen Anlagen durch zweijährige Kultur auf Beet $A$, teils aber auch der Umstand, daß die Erde zur Hälfte aus lockerer, nährstoffreicher Mistbeeterde bestand, eine weitere Abnahme verhindert.

Auch über die Wirkung der verschiedenen Nährsalze kann ich noch keine bestimmten Angaben machen. Nach den oben mitgeteilten Versuchen, darf man aber wohl annehmen, daß manche künstlichen Düngemittel einen günstigen Einfluß auf die Füllung ausüben. Sie nahm bei Nr. 11, die $2 \mathrm{~g}$ Kalkstickstoff erhalten hatte $1 \mathrm{p}$ und 2 $\mu \top \mathrm{zu}$, während Nr. 28 mit $1 \mathrm{~g}$ Chilisalpeter gedüngt nur $1 \mathrm{p}$ mehr als im Jahre vorher hatte. Im Jahre 1905 hatte bei Nr. 13 (alt numeriert) (mit $1 \mathrm{~g}$ Chilisalpeter) die Gesamtzahl der Blattgebilde zwar nicht zugenommen aber $3 \mu \mathrm{T}$ hatten sich in $3 \mathrm{p}$ verwandelt, was immerhin als eine nicht unbedeutende Zunahme anzusehen ist. Nr. 127 hatte allerdings, troz Düngung mit $1 \mathrm{~g}$ Chilisalpeter in der Füllung abgenommen. Nr. $115 \mathrm{nahm}$ bei einer Mischung aus $1 \mathrm{~g}$ schwefelsaurem Ammoniak, $1 \mathrm{~g}$ schwefelsaurem Kali und $1 \mathrm{~g}$ Superphosphat in der Füllung der Blüte und zwar nicht unbedeutend, zu. Nr. 116 hatte bei Dünguug mit $1 \mathrm{~g}$ schwefelsaurem Ammoniak $1 \mathrm{p}$ mehr, denn die übrige Verschiebung $1 \mu \mathrm{T}$ und $3 \mu \Gamma$, statt $2 \mu \top$ und $1 \mu \Gamma$ gleicht sich aus. Voßsches Nährsalz Nr. 4 bewirkte bei Nr. 20 eine Zunahme, wogegen Nr. 136 um $2 \mu \top$ und $4 \mu \Gamma$ abnahm, weswegen sich hier noch kein Schluß ziehen läßt, umsoweniger als diese beiden in einem Topfe standen. Dagegen trat bei $97 \mathrm{e}$, a, die mit $\check{\rho} \mathrm{g}$ Wag. Nährsalz ${ }^{1}$ ) gedüngt wurde, eine bedeutende Abnahme von $5 \mu \Gamma$ ein. Es ist aber zu berücksichtigen, daß sie in Komposterde und im Jahre vorher auf Beet $\mathrm{F}$ stand. Da aber auch die Füllung von Nr. 13 (alt numeriert) im Jahre 1906 bei 5 g Kalkstickstoffdüngung, trotz vorausgegangener günstiger Kultur und Zunahme sehr abnahm - für den Verlust von $5 \mathrm{p}$ waren nur $1 \mu \mathrm{T}$ und $1 \mu \Gamma$ hinzugekommen, auch Nr. 22a bei Düngung mit $63 / 4$ Thomasphosphatmehl $1 \mathrm{p}$ und $1 \mu \Gamma$

1) Gemeint ist damit das von Prof. Wagner 1893 in seiner Broschüre „Die Anwendung künstlicher Düngemittel" pag. 33 empfohlene Nährsalz. 
weniger als ihre Schwesterzwiebel hatte, liegt der Schluß nahe, daß sehr starke künstliche Düngungen einen entgegengesetzten Einfluß haben als mäßige, so dảß sie die Füllung vermindern, statt sie zu stärken. Dies läßt besonders der Vergleich mit der günstigen Wirkung von $2 \mathrm{~g}$ Kalkstickstoff wahrscheinlich erscheinen. Danach wäre wohl die geeignetste Quantität, soweit es sich bis jetzt beurteilen läßt, 2 , höchstens $3 \mathrm{~g}$ für einen $13 \mathrm{~cm}$ großen Topf, denn $1 \mathrm{~g}$ schien nur wenig und mehr als $3 \mathrm{~g}$ einen ungünstigen Einfluß zu haben.

Beim Treiben auf Wasser ist ein noch viel geringeres Quantum $\mathrm{zu}$ verwenden, denn $1 \mathrm{~g}$ Wagnersches Nährsalz wirkte pro $1 / 2$ LiterGlas schon zu stark, wie wir bei Nr. 23 sehen, deren Petala zwar an Zahl zugenommen hatten, aber alle etwas schmal waren. Diese $\mathrm{Zu}$ nahme wurde auch durch unverhältnismäßigen Rückgang der Übergangsgebilde mehr als ausgeglichen und ferner ging die Zwiebel nach dem Treiben kaputt. Es ist deshalb am besten von Nährsalz nur $1 / 2 \mathrm{~g}$ pro $1 / 2$ Liter-Glas anzuwenden.

Es scheint mir aus den Topfkulturen hervorzugehen, daß der günstige Einfluß mancher Nährsalze, vor allem - wenn nicht ausschließlich - auf Rechnung des Stickstoffs kommt, da nur dieser in allen die Füllung stärkenden Nährsalzen und Düngesalzen enthalten war, diese aber im übrigen von einander abwichen. 